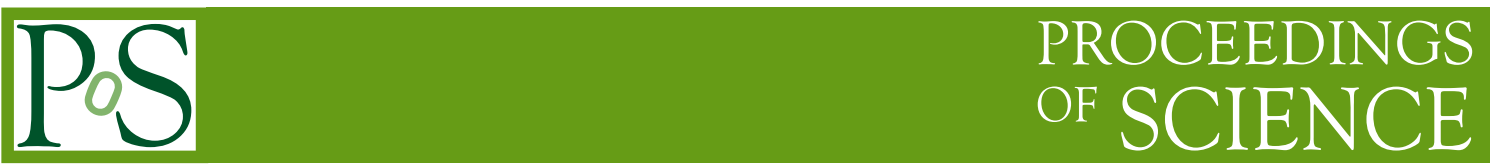

\title{
Multifrequency Behaviour of Mrk 509
}

\author{
Rozenn Boissay ${ }^{* \dagger}$ \\ Department of Astronomy, University of Geneva - Switzerland \\ E-mail: rozenn.boissayaunige.ch
}

\begin{abstract}
Active galactic nuclei (AGN) are the most luminous persistent objects in the universe. The Xray domain is particularly important because the X-ray flux represents a significant fraction of the bolometric emission from such objects and probes the innermost regions of accretion disks, where most of this power is generated. An excess of X-ray emission below $\sim 2 \mathrm{keV}$, called soft-excess, is very common in Type 1 AGN spectra. The origin of this feature remains debated. Originally modelled with a blackbody, there are now several possibilities to model the soft-excess, including warm Comptonization and blurred ionized reflection.

Mrk 509 is a bright Seyfert 1 galaxy that has been observed during a multiwavelength campaign in 2011, using five satellites (XMM-Newton, INTEGRAL, Chandra, HST and Swift) and two ground-based facilities (WHT and PAIRITEL). I will present the results of Mehdipour et al. (2011), Petrucci et al. (2013) and Boissay et al. (2014), studying the soft-excess of Mrk 509 based on data from the multiwavelength campaign. Warm Comptonization seems to be the most probable origin of the soft-excess in this object. Nevertheless, it is possible that both ionized reflection and warm Comptonization mechanisms coexist, one mechanism dominating the other one and explaining the soft-excess, depending on physical conditions of the disk and the corona.
\end{abstract}

XI Multifrequency Behaviour of High Energy Cosmic Sources Workshop

25-30 May 2015

Palermo, Italy

* Speaker.

On behalf of the Mrk 509 consortium. 


\section{Introduction}

The X-ray spectrum of Seyfert galaxies is typically characterized by a power-law continuum with reflection features, absorption, and often an excess in the soft X-rays. The power-law emission is believed to be due to the Comptonization of the UV photons coming from the disk by the energetic electrons in a corona surrounding the disk, whose structure is still debated. The strongest signatures of the reflection component are a "reflection hump" around $30 \mathrm{keV}$ and an iron FeK $\alpha$ fluorescence line in between 6 and $7 \mathrm{keV}$ depending on the iron ionization state. Reflection is associated with the reprocessing of the primary continuum by material either close to the central black hole, in the accretion disk, in a torus, or in the narrow- or broad-line regions. Absorption is generally observed in the X-ray spectra of Seyfert 1 and 2 galaxies. If the absorbing material is photoionized, it is referred to as "warm absorber".

A soft X-ray emission in excess of the extrapolation of the hard X-ray continuum is detected in many Seyfert $1 \mathrm{~s}$ below $2 \mathrm{keV}$. This feature, the soft-excess (SE), is almost ubiquitous in Seyfert 1s. The soft-excess was first believed to originate in the inner part of the accretion flow and it has been modelled for a long time using a blackbody (kT $\sim 0.1-0.2 \mathrm{keV}$ ). A possible explanation for the soft-excess is the "warm" Comptonization scenario: UV seed photons from the disk are upscattered by a Comptonizing corona cooler and optically thicker than the hot corona responsible for the primary X-ray emission. This model has been successfully applied for example to NGC 5548 (Magdziarz et al. 1998) and Ark 120 (Matt et al. 2014). This Comptonization model is supported by the existence of similarities between the spectral shape (Walter \& Fink 1993) and the variability of the optical/UV and soft X-ray emission (Edelson et al. 1996). Another interpretation is that the soft-excess is the result of ionized reflection in a relativistic disk, which blurs all emission lines. Crummy et al. (2006) successfully applied such an ionized-reflection model on a sample of PG quasars. Time delays between iron lines and direct X-ray continuum have been detected in several objects (e.g. 1H 0707-495, Fabian et al. 2009; Ark 564 and Mrk 335, Kara et al. 2013) as well as soft X-ray reverberation lags (e.g. De Marco et al. 2013). We will show here that the multifrequency campaign of Mrk 509 is a powerful tool to determine the origin of the soft-excess in this object.

\section{Mrk 509 - multiwavelength campaign}

Mrk 509 is a bright Seyfert 1 galaxy, which presents a strong soft-excess below $2 \mathrm{keV}$ and a resolved iron line at $6.4 \mathrm{keV}$. This object has been observed during a multiwavelength campaign presented in Kaastra et al. (2011), using five satellites, XMM-Newton, INTEGRAL, Chandra, HST, and Swift, and two ground-based facilities (WHT and PAIRITEL). This multifrequency campaign allows to study Mrk 509 spectra on a wide energy range, and the multiple and simultaneous observations, especially with XMM-Newton and INTEGRAL, enable us to study its variability.

\section{Soft-excess: the warm Comptonization hypothesis}

\subsection{Correlation optical-UV / soft X-ray emission}

During the unprecedented multiwavelength campaign presented above, Mrk 509 has been ob- 

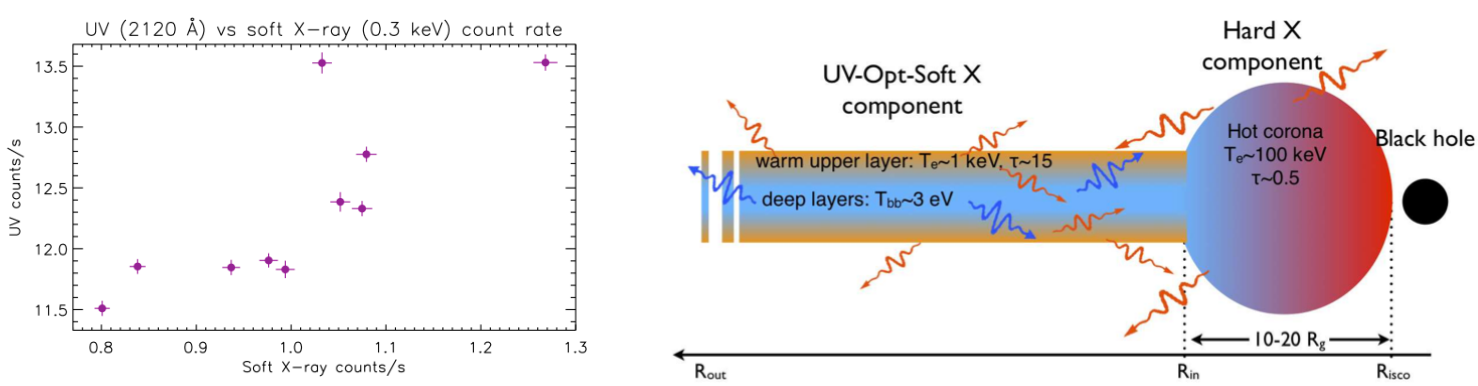

Figure 1: Left panel: the UV count rate from the OM UVW2 filter plotted versus the EPIC-pn soft X-ray count rate for the 10 XMM-Newton observations of the Mrk 509 campaign (Mehdipour et al. 2011). Right panel: a sketch of the accretion flow geometry in the inner region of the galaxy Mrk 509 (Petrucci et al. 2013).

served by the XMM-Newton satellite, which provided ten observations of about $60 \mathrm{ks}$ each, separated by four days. Mrk 509 has also been monitored by Swift before and after the XMM-Newton observations. Mehdipour et al. (2011) used these data, as well as HST/COS and FUSE observations, to establish the continuum SED in the optical-UV and X-ray bands and to investigate its variability on the timescale of the campaign with a time resolution of a few days. They found the presence of a soft X-ray emission in excess of the extrapolation of the X-ray power-law below $2 \mathrm{keV}$. On one hand, they noted that this soft-excess varies in association with the thermal opticalUV emission from the accretion disk (see the correlation between fluxes in the optical-UV and soft X-rays bands in left panel of Fig. 1). Such a correlation is expected if the soft-excess is due to warm Comptonization of the seed photons from the disk. On the other hand, they found that the flux variability of the power-law component is uncorrelated with the variability of the soft-excess and the disk emission. The results of their simultaneous broad-band spectral and timing analysis suggest that, on a time scale of a few days, the soft X-ray excess of Mrk 509 is produced by the Comptonization of the thermal optical-UV photons from the accretion disk by a warm $(0.2 \mathrm{keV})$ optically thick ( $\tau \sim 17$ ) corona surrounding the inner regions of the disk.

\subsection{Testing warm Comptonization model}

Petrucci et al. (2013) used the ten simultaneous XMM-Newton and INTEGRAL observations, as well as HST/COS and FUSE data, to fit the average broad-band energy spectrum (from UV to X-ray/gamma-ray emission) with a realistic thermal Comptonization model for the continuum emission, including the Galactic absorption, a warm absorber and a neutral reflection component (Detmers et al. 2011, Ponti et al. 2013), and adding a warm Comptonization component to model the soft-excess (Mehdipour et al. 2011). Using XSPEC, they modelled the primary continuum with a compps model, the soft-excess with nthcomp, and the neutral reflection component with pexmon. They found that the primary continuum is produced by a hot $(\mathrm{kT} \sim 100 \mathrm{keV})$, opticallythin $(\tau \sim 0.5)$ corona, while the soft-excess component requires a warm $(\mathrm{kT} \sim 1 \mathrm{keV})$, opticallythick ( $\tau \sim 10-20)$ plasma. They proposed a geometry where this warm plasma is the upper layer of the accretion disk (see right panel of Fig. 1). In this sketch, the hot corona, producing the hard $\mathrm{X}$-ray emission, is photon-starved, localized in the inner part of the accretion flow, and illuminates 
the accretion disk beyond $R_{i n}$, helping to form a warm layer at the disk surface. This warm component, which extends over a large part of the accretion flow, heats the deeper layers of the disk and comptonizes their optical-UV emission to produce the soft-excess. In return, part of this emission participates in the cooling of the hot corona.

\section{Soft-excess: the blurred ionized-reflection hypothesis}

We have seen that, using observations from the multiwavelength campaign of Mrk 509, Mehdipour et al. (2011) and Petrucci et al. (2013) explain efficiently the soft-excess in Mrk 509 with a warm Comptonization scenario. But can we rule out the hypothesis of blurred ionized reflection as the origin of the soft-excess in Mrk 509?

\subsection{Model and parameters}

In XSPEC, Boissay et al. (2014) used a blurred ionized reflection model to fit each of the ten XMM-Newton and INTEGRAL observations, taking into account absorption (Detmers et al. 2011). The primary continuum has been fitted by a simple cut-off power-law (cutoffpl), and the neutral reflection, similarly to Petrucci et al. (2013), is modelled by pexmon. To model the soft-excess, Boissay et al. (2014) convolved the ionized-reflection model reflionx with the Laor model shape kdblur. By fitting the data, they obtained a very strong reflection factor of $R \sim 5$ which underestimates data at high energy, and extreme parameters and geometries (the emissivity index and the inner radius reaching the model limits). They also obtained low ionization parameters (compared to results from Crummy et al. 2006) needed to reproduce the soft-excess, but enable to produce the Fe XXV and Fe XXVI lines found in Mrk 509 by Ponti et al. (2013). Their fitting resulted in less satisfactory chi-square statistics and stronger residuals around the iron line compared to the ones obtained when fitting with a warm Comptonization model.

\subsection{Long time scale variability of the soft-excess}

Boissay et al. (2014) plotted the rms fractional variability as a function of the energy (see left panel of Fig. 2) on short time scale (1 ks - $60 \mathrm{ks}$, black points) and on long time scale (60 ks - 1 month, red points). They noticed the same decrease for the hard X-ray emission parts on different time scales and a decoupling between the soft and the hard emission variability, with a maximum variability at low energy for $\mathrm{T}>60 \mathrm{ks}$.

In the hypothesis of a lamp-post configuration, where a primary source of X-rays is located above the accretion disk close to a central black hole and illuminates both the observer at infinity and the accretion disk (Miniutti \& Fabian 2004), the light bending effect increases when the height of the source decreases, implying a higher reflection factor $R$. In this configuration, the reflection is expected to vary less than the power-law. The light bending effect present in the lamp-post configuration could then explain the variability profile on short time scale, as the maximum variability is found where the power-law dominates.

This hypothesis of lamp-post configuration does not hold anymore when considering the variability on longer time scale. In the hypothesis of blurred ionized reflection, could this variability of the soft-excess be due to slow changes of the ionization? As the ionization parameter is defined by $\xi=\frac{L}{n r^{2}} \operatorname{erg~cms~}^{-1}$, it decreases for an increasing density $n$. On the other hand, an increasing 

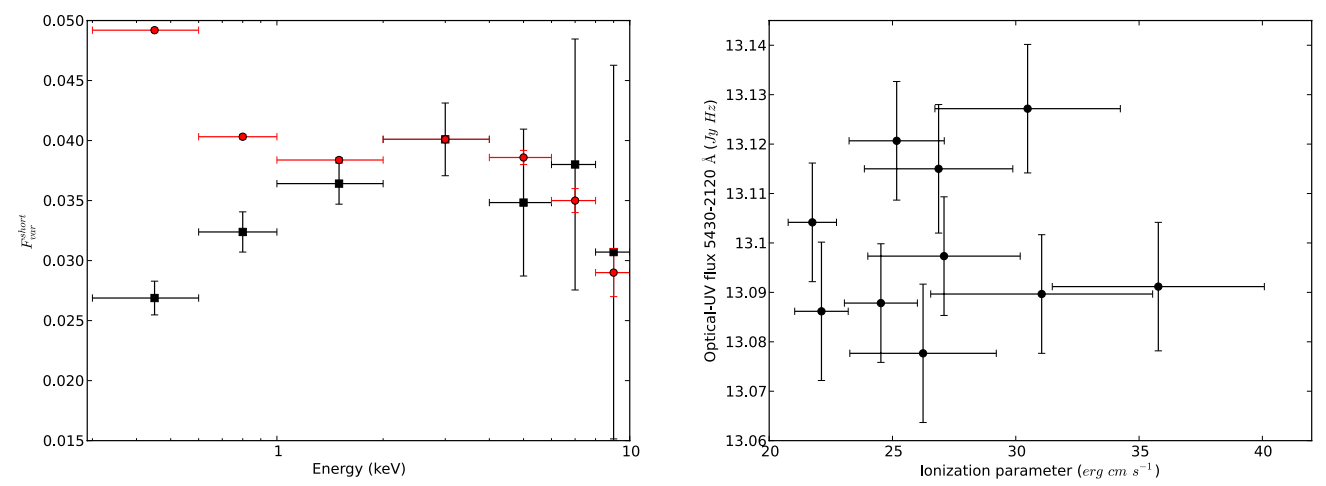

Figure 2: Left panel: rms fractional variability as a function of energy. The black squares represent the variability spectrum on short time scale $(1 \mathrm{ks} \lesssim \mathrm{T} \lesssim 60 \mathrm{ks})$; the red circles are the corresponding variability values predicted from the long time scale variability spectrum ( $60 \mathrm{ks} \lesssim \mathrm{T} \lesssim 1$ month). Right panel: opticalUV flux as function of the ionization parameter (Boissay et al. 2014).

$n$ implies a more important accretion rate, hence a larger UV flux. An anti-correlation between the UV flux and the ionization parameter is then expected in this case, but it is not verified by the data (see right panel of Fig. 2). The long term variations of the soft-excess can not be attributed to density changes.

\subsection{Evolution of ionized-reflection model parameters for different geometries}

Boissay et al. (2014) studied the evolution of fitted parameters for two different geometries: a stable geometry and a lamp-post configuration. In the hypothesis of a stable geometry (i.e. a constant $R$ ), it is shown on the left panel of Fig. 3 that the black data points do not follow the flat relation expected between the reflionx normalization and the ionization parameter in the case of blurred ionized reflection (solid red line). Indeed, a non-physical anti-correlation appears when fitting the ten observations, probably due to fine-tuning of reflionx parameters. This allows us to reject the hypothesis of a stable geometry.

A lamp-post configuration with $R$ variable is able to explain the anti-correlation found between $R$ and the power-law flux, as for a decreasing height of the source, the light bending effect will be more important and $R$ will increase, reducing the number of primary photons reaching the observer. In this case, a correlation between the height of the source and the reflection dominated continuum can be expected, as shown on Figure 1 from Miniutti \& Fabian (2004), as Mrk 509 shows a high reflection factor and a steep emissivity profile which correspond to a small value of the height. However, the fact that such a correlation is not found in the data (see right panel of Fig. 3) does not support the hypothesis of a lamp-post configuration.

\section{Conclusion}

Monitoring campaigns allow studying emission models along the time dimension and can provide very useful constraints to determine the origin of components like the soft-excess. The unique data set provided by the multiwavelength campaign of Mrk 509 enable, by broad-band 

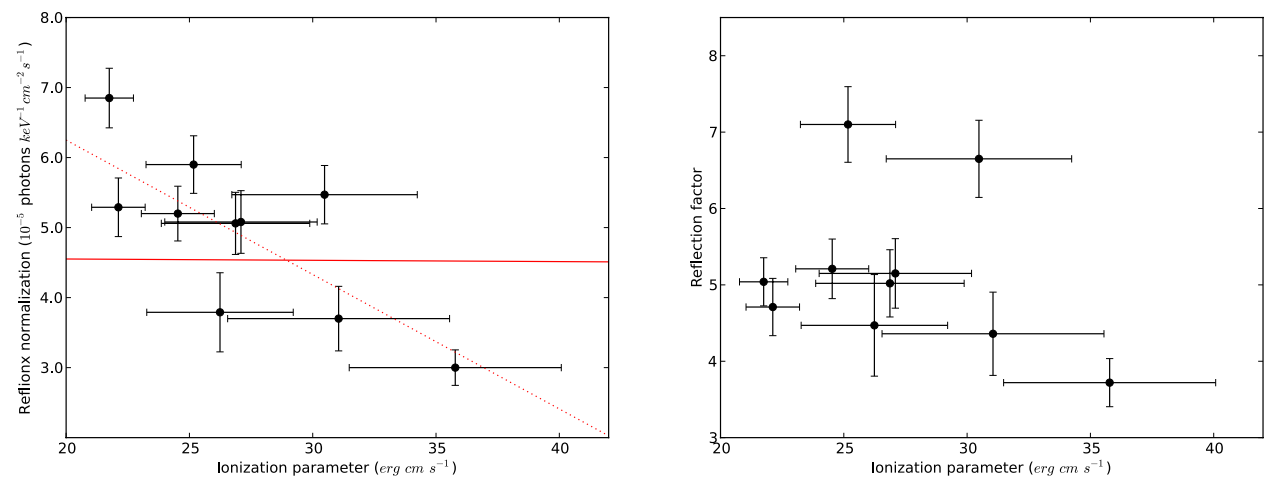

Figure 3: Left panel: reflionx normalization as a function of the ionization parameter $\xi$ (black points: data; solid red line: evolution expected with a reflionx model). Right panel: $R$ as a function of $\xi$.

fitting (from UV to gamma-rays) and variability study, to conclude that the most probable origin of the soft-excess in this object is warm Comptonization (Mehdipour et al. 2011, Petrucci et al. 2013), and to rule out the hypothesis of blurred ionized reflection, as the model tested by Boissay et al. (2014) seems unable to explain the soft-excess in Mrk 509 neither for a stable geometry, nor for a lamp-post configuration. Both mechanisms may be at work in all objects, one dominating over the other, depending on physical conditions of the disk and the corona. The advent of sensitive telescopes such as ASTRO-H will provide better constraints on the origin of the soft-excess.

\section{References}

[1] Boissay, R., Paltani, S., Ponti, G., et al. 2014, A\&A, 567, A44

[2] Crummy, J., Fabian, A. C., Gallo, L., \& Ross, R. R. 2006, MNRAS, 365, 1067

[3] De Marco, B., Ponti, G., Cappi, M., et al. 2013, MNRAS, 431, 2441

[4] Detmers, R. G., Kaastra, J. S., Steenbrugge, K. C., et al. 2011, A\&A, 534, A38

[5] Edelson, R. A., Alexander, T., Crenshaw, D. M., et al. 1996, ApJ, 470, 364

[6] Fabian, A. C., Zoghbi, A., Ross, R. R., et al. 2009, Nature, 459, 540

[7] Kaastra, J. S., Petrucci, P.-O., Cappi, M., et al. 2011, A\&A, 534, A36

[8] Kara, E., Fabian, A. C., Cackett, E. M., et al. 2013, MNRAS, 434, 1129

[9] Magdziarz, P., Blaes, O. M., Zdziarski, A. A., Johnson, W. N., \& Smith, D. A. 1998, MNRAS, 301, 179

[10] Matt, G., Marinucci, A., Guainazzi, M., et al. 2014, MNRAS, 439, 3016

[11] Mehdipour, M., Branduardi-Raymont, G., Kaastra, J. S., et al. 2011, A\&A, 534, A39

[12] Miniutti, G. \& Fabian, A. C. 2004, MNRAS, 349, 1435

[13] Petrucci, P.-O., Paltani, S., Malzac, J., et al. 2013, A\&A, 549, A73

[14] Ponti, G., Cappi, M., Costantini, E., et al. 2013, A\&A, 549, A72

[15] Walter, R. \& Fink, H. H. 1993, A\&A, 274, 105 